\section{Comparison of canine intravenous catheter site preparation with standard chlorhexidine gluconate and isopropyl alcohol scrub versus a commercial single-use $2 \%$ chlorhexidine gluconate and 70\% isopropyl alcohol applicator}

\section{Emily Brooke, Simon Tappin, Federico Corletto}

Dick White Referrals, Suffolk, UK

\section{OBJECTIVES}

To compare the efficacy in reducing bacterial counts of a standard skin preparation method and a commercial single-use applicator, prior to canine peripheral intravenous catheter placement. Single-use applicators have gained popularity recently, being more convenient and potentially quicker than standard scrub technique.

\section{METHODS}

Forty client-owned dogs were enrolled in a prospective randomised non-blinded study. In twenty dogs catheter placement site was prepared with standard chlorhexidine gluconate surgical scrub and isopropyl alcohol, in the other twenty dogs a commercial single-use applicator containing $2 \%$ chlorhexidine gluconate and $70 \%$ isopropyl alcohol was used. Exclusion criteria included antibiotics or corticosteroid use in the previous 10 days and prior catheter placement in the limb. The site was clipped and a swab taken prior to and after skin preparation using a standardised technique in both groups. All swabs underwent bacterial culture with bacterial count and the species recorded. D'Agostino Pearson normality test was used to assess whether variables were normally distributed. Age and weight were compared using a Student's t test, gender using Fisher's exact test, and treatments using a two-way (treatment and time) ANOVA.

\section{RESULTS}

No significant differences were found between groups for age, weight, and gender. Bacterial species identified were similar to previous studies, including Staph. spp and Acinetobacter calcoaceticus. Ability to reduce bacterial count was similar between groups $(p=0.67)$, with both techniques showing a significant reduction in bacterial counts $(P=0.003)$.

\section{STATEMENT}

Both skin preparation methods are suitable for use prior to catheter placement and are likely to reduce the risk of catheter associated infection and complications. 